\title{
México: ¿Cómo inciden las políticas monetarias en las tasas de desempleo?
}

Alejandro Islas C. y Willy Walter Cortez

RESUMEN

En este artículo se analiza el impacto de un choque (shock) de política monetaria en las tasas de desempleo de México. A diferencia de estudios anteriores, en este se volvieron a calcular las tasas de desempleo para compararlas con las de los países de la Organización de Cooperación y Desarrollo Económicos (OCDE). La conclusión es que, ante una política monetaria restrictiva, el desempleo aumenta siguiendo el mismo patrón en forma de $U$ invertida que se observa en otros estudios. Los resultados son robustos con respecto a diferentes supuestos sobre la naturaleza del mercado laboral mexicano. de México (ITAM). aislas@itam.mx

Willy Walter Cortez es coordinador de maestría en economía del Departamento de Métodos Cuantitativos de la Universidad de Guadalajara. wcortez@cucea.udg.mx 


\section{I}

\section{Introducción}

Desde finales de los años cincuenta y principios de la década de 1960, la inflación se ha convertido en una de las mayores preocupaciones tanto en países industrializados como en los de economías emergentes. Friedman (1976) ilustró la repercusión negativa de la inflación alta y de la volatilidad de la inflación en los resultados económicos. La inflación alta, por ejemplo, puede reducir la cohesión política al dejar sin efecto los acuerdos institucionales y los contratos financieros basados en un nivel de precios "normal" a largo plazo. Por la incertidumbre que genera, la alta volatilidad de los precios puede disminuir la eficiencia económica y tornar los precios de mercado en un sistema de coordinación de la actividad productiva menos eficiente. Debido a todos estos efectos negativos en la expansión económica, los bancos centrales suelen considerar a la inflación como el mayor problema que un país debe enfrentar.

En el caso de México, la evidencia empírica del impacto negativo de la inflación en el crecimiento es de alguna manera mixta. Por ejemplo, en un estudio reciente, Grier y Grier (2006) estimaron que la incertidumbre causada por la inflación había tenido un efecto negativo y significativo en el producto de México. Por otra parte, detectaron que aumentos en las tasas medias de inflación habían provocado mayor incertidumbre, lo que a su vez afectó a la expansión de la economía. No obstante, Risso y Sánchez (2009) afirman que la inflación tiene un efecto positivo en el crecimiento cuando se mantiene bajo el umbral del $9 \%$, en tanto que las tasas de inflación que sobrepasan este umbral lo afectan negativamente.

A todas luces, la política monetaria de México ha logrado en los últimos 25 años alcanzar la estabilidad de precios: la inflación disminuyó de una tasa media mensual del 4,3\% durante la década de 1980 a un $0,4 \%$ durante los primeros años del siglo XXI. Las autoridades monetarias mexicanas han utilizado varios instrumentos para lograr la estabilidad de precios, desde controles cambiarios hasta el control de la base monetaria y políticas de metas inflacionarias. A fin de lograr esto último, el Banco de México ha mantenido una política de control de la cantidad de moneda disponible para los bancos comerciales por medio de los saldos que mantienen con el banco central ${ }^{1}$, lo que

${ }^{1}$ El Banco de México es la institución que corresponde al banco central del país. provoca cambios en las tasas de interés internas. A modo de ejemplo, las tasas de interés más altas han generado contracciones de la demanda agregada que aminoran la inflación interna. Asimismo, en una economía abierta con gran movilidad de capital y tipos de cambio flexibles, las tasas de interés internas más elevadas disminuyen aún más la tasa de inflación por medio de la apreciación de la moneda local: una moneda local más fuerte no solo frena las exportaciones, sino que también reduce los precios internos de los productos importados y aumenta los precios de los productos exportables.

En los estudios empíricos de la neutralidad de la política monetaria de México se presentan pruebas contradictorias. Por ejemplo, Mendoza (2003) llega a la conclusión de que en México no hay una correspondencia a largo plazo entre la volatilidad del producto y la de la inflación, de manera que la política monetaria solo afecta a los precios en el largo plazo. En cambio, Galindo y Ros (2005) concluyen que la política monetaria sí ha tenido cierto efecto en el sector real.

Si bien se ha reconocido la repercusión negativa que la política monetaria mexicana de ajuste tuvo en la demanda agregada ${ }^{2}$, hasta ahora no se ha realizado un análisis integral del efecto de esta política en el mercado laboral. Esto constituye una gran deficiencia de la agenda de investigación actual de México, dado que ello significa que se pasa por alto el costo de lograr la estabilidad de precios en términos del bienestar de los trabajadores. Incluso si se reconoce que la estabilidad de precios es una condición necesaria para el crecimiento económico a largo plazo, no se deben desconocer los costos de corto y mediano plazo para el bienestar de los trabajadores.

En este trabajo se investiga con mayor profundidad cómo la política monetaria incide en el desempleo en México, un país que comenzó su exitoso programa de estabilización de precios durante la segunda mitad de la década de 1980. En relación con esto cabe preguntarse cuán permanente es la repercusión de la política monetaria en la tasa de desempleo.

El modelo utilizado para llevar a cabo los análisis empíricos se basa en el supuesto de que el dinero no

\footnotetext{
2 Véase, por ejemplo, Díaz de León y Greenham (2000).
} 
es neutral; es decir, se sostiene que los cambios en las tasas de desempleo pueden explicarse parcialmente por la política monetaria aplicada para controlar la inflación. El argumento central de este estudio es que al afectar a la demanda agregada — y, en consecuencia, al productola política monetaria tiene cierto efecto en las tasas de desempleo. El análisis comprende la estimación de un modelo de vectores autorregresivos estructurales (SVAR). La descomposición de la varianza ilustra cuáles fueron las perturbaciones (shocks) que causaron movimientos de las variables durante el período de análisis, mientras que las funciones impulso-respuesta contienen información sobre la magnitud y duración del efecto de una perturbación estructural determinada.

Otra característica distintiva del presente análisis es que se considera la tasa de desempleo oficial, así como una tasa alternativa de desempleo sobre la base de la definición utilizada por la Encuesta Continua de Población de la Oficina de Estadísticas Laborales de los Estados Unidos de América. Esta última tasa se utiliza en respuesta a algunas críticas surgidas contra la tasa de desempleo oficial.

Los resultados de este análisis indican que cuando se utiliza la tasa de desempleo alternativa, el ajuste de la política monetaria aumenta el desempleo siguiendo el característico patrón de U invertida que se muestra en Alexius y Holmlund (2007) y Ravn y Simonelli (2008). La tasa de desempleo alcanza su nivel máximo después del segundo trimestre, cuando registra 0,26 puntos sobre su nivel original, y luego vuelve gradualmente a ese nivel inicial. Los resultados de la descomposición de la varianza del error señalan que el shock de política monetaria es responsable del $3 \%$ al $27 \%$ de la fluctuación en el desempleo. En cambio, cuando se utiliza la tasa de desempleo oficial las repercusiones de política monetaria no tienen demasiado efecto.

El presente trabajo está organizado según se describe a continuación. En la sección II se presenta un breve análisis de la no neutralidad del dinero y se describe cómo la política monetaria puede afectar al desempleo. En la sección III se examinan las políticas monetarias implementadas por las autoridades mexicanas durante el período de análisis, mientras que en la sección IV se presentan algunas características clave del mercado laboral mexicano y se introduce un indicador alternativo de las tasas de desempleo. En la sección V se presenta la metodología utilizada para efectuar el análisis empírico. En la sección VI se incluyen los principales resultados empíricos y en la sección VII se presentan las conclusiones.

\section{II}

\section{¿Es neutral el dinero?}

Las discusiones sobre la neutralidad monetaria se remontan al ensayo de Cantillon (1775) publicado a mediados del siglo XVIII. Su argumento era que, bajo el patrón oro, los incrementos de la oferta de dinero fomentarían un mayor gasto, lo que a su vez estimularía la producción. Sin embargo, este efecto positivo en la actividad productiva sería poco duradero, puesto que la mayor demanda generaría precios más altos, lo que afectaría negativamente a la producción. El mismo argumento se encuentra en el trabajo de Hume (1985) sobre el dinero. Sus puntos de vista fueron reformulados por Newcomb (1885) y Fisher (1911), y se convirtieron en lo que actualmente se conoce como la teoría cuantitativa del dinero.

Por otra parte, la no neutralidad del dinero es una característica clave en la economía keynesiana. La postura de Keynes (2001) sobre la repercusión del dinero en las variables reales se encuentra en su Teoría general de la ocupación, el interés y el dinero. El punto central de su argumento es el papel que juegan las tasas de interés internas en los cambios de la demanda agregada. En el marco keynesiano, la tasa de interés es la recompensa por privarse de la liquidez. En la medida en que la tasa de interés afecte a la eficiencia marginal del capital, que a su vez determina la inversión, los cambios en la cantidad de dinero afectarán al producto y el empleo. El impacto general en la demanda agregada se ve incrementado por la sensibilidad del consumo a los cambios en la inversión. Según Keynes, la tasa de interés depende, en parte, del estado de las preferencias por la liquidez y, en parte, de la cantidad de dinero, de manera tal que los cambios en la cantidad de dinero provocarán cambios en la tasa de interés, ceteris paribus. A su vez, Chick (1983) sostiene que las tasas de interés se verán afectadas por las operaciones de mercado abierto o por las variaciones de las reservas bancarias o por el encaje bancario, diseñado para afectar a la liquidez o a la oferta de crédito, o a ambas. 
Dada la capacidad de las tasas de interés para afectar al producto y, en consecuencia, al desempleo, la autoridad monetaria puede utilizar la política monetaria para provocar cambios en la tasa de interés interna con el objetivo de lograr ciertas metas específicas. Si, por ejemplo, se asume que la inflación es causada por un exceso de demanda, entonces se pueden utilizar tasas de interés más altas para controlar la inflación por medio de la contracción de la demanda agregada. Asimismo, en una economía abierta con gran movilidad de capital, las tasas de interés más elevadas impulsarán la apreciación de la moneda local, lo que no solo frenará las alzas de precios, sino que también reducirá aún más la demanda agregada.

Por otra parte, Taylor (1994) sugiere que los esfuerzos de la autoridad monetaria por mantener los precios estables también pueden ocasionar una mayor fluctuación de la producción, mientras que los intentos de estabilizar el ciclo de producción pueden generar una mayor volatilidad de los precios. A fin de reducir dicha volatilidad, la política monetaria tendría que ser más sesgada a la restricción de la demanda agregada.

En general, existe consenso acerca de la no neutralidad del dinero en el corto plazo. No obstante, recientemente se ha generado un creciente debate sobre la repercusión a largo plazo de la política monetaria en el sector real. Blanchard (2003), por ejemplo, afirma que el dinero no es neutral y que la política monetaria puede tener efectos importantes y prolongados en las tasas de interés y, en consecuencia, en el desempleo. Existen tres canales posibles de transmisión. Uno podría ser el efecto de la tasa de interés real en el desempleo actual. Cuanto más prolongado sea el período de desempleo, mayor será la probabilidad de que los desempleados pierdan sus habilidades. Por lo tanto, un desempleo alto y sostenido conducirá a un aumento de la propia tasa natural. El segundo canal posible es la acumulación de capital: la tasa de interés real afecta al costo de capital, el costo de capital afecta a la acumulación de capital y la acumulación de capital afecta a la demanda de mano de obra y, en consecuencia, al desempleo. Por consiguiente, un incremento sostenido de las tasas de interés real provoca primero un aumento de la tasa de desempleo real y, luego, a medida que la acumulación de capital disminuye, un incremento de la tasa natural. El tercer mecanismo posible puede ser el efecto de la tasa de interés real en el margen de beneficio de una empresa. En la medida en que esto tenga un efecto directo en el nivel de empleo, el desempleo se verá afectado por la política monetaria a través de este mecanismo ${ }^{3}$.

La teoría de la neutralidad del dinero a largo plazo predice que una política monetaria de ajuste no afectará a la trayectoria de crecimiento; sin embargo, Schettkat y Sun (2008) explican que en Europa, por ejemplo, la política monetaria ha sido asimétrica debido al temor a la inflación de los bancos centrales. Estos autores presentan pruebas de que esta asimetría ha reducido la tendencia del crecimiento a largo plazo en algunos países europeos ${ }^{4}$. En otros estudios, donde también se ha hallado evidencia en contra de la neutralidad del dinero a largo plazo, se confirma este resultado.

En cierta medida, esta asimetría de la política monetaria es una característica intrínseca de la política de metas inflacionarias. Los estudios sobre la repercusión de la política monetaria asimétrica en el producto en países menos desarrollados son poco comunes. Uno de los escasos que existen es el de Galindo y Ros (2005). Estos autores sostienen que la política monetaria en México ha estado sesgada a la apreciación del tipo de cambio desde el inicio de la política de metas inflacionarias, es decir, el banco central ha respondido únicamente cuando el tipo de cambio ha estado subvalorado, y no cuando el tipo de cambio real estuvo sobrevalorado. Asimismo, el efecto negativo en el producto de la apreciación del tipo de cambio real significa que la política monetaria (o la política de metas inflacionarias) ha frenado el crecimiento económico de México.

Una posible conclusión que se puede desprender de este análisis es que existen sólidos argumentos teóricos en favor no solo de la no neutralidad del dinero a corto plazo, sino también a largo plazo. La cuestión giraría entonces en torno de si la política monetaria en México ha tenido una repercusión de corto y largo plazo en el desempleo. En la próxima sección se describirán brevemente las políticas monetarias y fiscales aplicadas en el país durante el período comprendido entre 1987 y 2004.

\footnotetext{
${ }^{3}$ Existe un cuarto mecanismo potencial que está comprendido dentro del enfoque del ciclo económico real: la oferta de mano de obra depende de las tasas de interés. Esta es un área que no ha sido investigada todavía con respecto a las economías menos desarrolladas. ${ }^{4}$ Schettkat y Sun (2008) ofrecen la siguiente explicación. Una perturbación negativa externa empuja a la economía a una recesión que no se ve totalmente contrarrestada por la política monetaria. Durante la reactivación, por temor a la inflación, el banco central no se adapta completamente a la recuperación. Como resultado, la economía no volverá a su trayectoria de crecimiento original, sino que se mantendrá por debajo de ella.
} 


\section{III}

\section{Las políticas monetarias y fiscales en México}

El presente análisis comienza en 1987, en un período caracterizado por una gran inestabilidad macroeconómica. En esa época la política monetaria de México tenía el doble objetivo de promover el crecimiento económico junto con la estabilidad de precios (Garriga, 2010). Una característica distintiva de esa política monetaria fue que con ella se intentó controlar la inflación por medio de la estabilidad cambiaria. Messmacher y Werner (2002) afirman que las autoridades mexicanas utilizaron varios instrumentos para controlar la inflación, entre los que se destacaron los instrumentos fiscales junto con los controles cambiarios. Además, el gobierno dispuso el congelamiento temporal de los salarios y aplicó controles de precios como parte de un Pacto de Solidaridad Económica.

El uso de estos instrumentos se explica por la premisa de que la inflación se debe principalmente a los déficits fiscales que derivaron en el aumento de la demanda agregada en relación con la oferta agregada. En este contexto, el exceso de demanda se consideró no solo como conducente a precios internos más altos, sino también a déficits de cuenta corriente. A su vez, los déficits externos crearían presión para la devaluación del tipo de cambio, lo que podría exacerbar aún más la inflación (Garriga, 2010).

A medida que se iban levantando la mayoría de los controles de precios y salarios, el programa antiinflacionario evolucionó hacia una estrategia más ortodoxa en la que el ajuste fiscal y el anclaje cambiario se convirtieron en los elementos principales de la política. Entre 1988 y 1994 las autoridades mexicanas efectuaron varias modificaciones al régimen cambiario y se reemplazó el régimen dual y fijo por una tasa de devaluación preanunciada (donde la tasa de devaluación se fijaba por debajo de la tasa de inflación). En noviembre de 1991, el Banco de México aplicó bandas cambiarias estrechas con un techo deslizable (Messmacher y Werner, 2002). Entre enero 1992 y octubre de 1993, el tipo de cambio nominal se mantuvo extraordinariamente estable. En diciembre de 1993, el Honorable Congreso de la Unión le otorgó autonomía operativa al Banco de México y estableció que su único objetivo era preservar la estabilidad del poder adquisitivo de la moneda, en reemplazo del doble objetivo que había tenido hasta entonces.
Durante la mayor parte de este período, el régimen cambiario administrado de México contaba con el apoyo de una política fiscal prudente. En efecto, el país pasó de un déficit fiscal del $12,5 \%$ del producto interno bruto (PIB) en 1988 a un superávit fiscal en 1992 (Garriga, 2010). Tras 18 meses, esta combinación de políticas produjo los resultados esperados: la inflación cayó pronunciadamente a menos del $20 \%$ anual. No obstante, a partir de ese momento el ritmo de reducción de la inflación se tornó lento.

Sánchez (2005) sostiene que entre 1988 y 1994 el volumen de crédito orientado al sector privado creció considerablemente, con tasas de incremento anuales cercanas al 30\%. La ausencia de un adecuado marco regulatorio y de supervisión, junto con normas deficientes de contabilidad y un reconocimiento insuficiente de préstamos anteriores vencidos, entre otros factores, significó que esta explosión del crédito afectara en forma negativa a la rentabilidad del sistema bancario. En conjunción con la falta de consonancia entre las políticas monetaria y cambiaria, los problemas del sistema bancario precipitaron una crisis muy importante a fines de 1994.

En diciembre de 1994, frente a una grave crisis financiera y de balanza de pagos, el Banco de México ya no pudo defender la paridad predeterminada del peso con respecto al dólar estadounidense y permitió la flotación del peso. Este último se depreció inmediatamente y los precios internos subieron a un ritmo constante, aumentando a una tasa mensual del 3,6\% a lo largo de 1995. La tasa de interés interbancaria subió de un $17 \%$ en la tercera semana de agosto de 1994 al $110 \%$ en la tercera semana de marzo de 1995, lo que provocó una grave depresión de la economía real. El producto y el empleo se vieron notablemente afectados: el producto cayó un 6,2\%, mientras que el desempleo alcanzó al $7,6 \%$ a mediados de 1995 . Sánchez (2005) señala que la recesión y el desempleo producto de la crisis financiera fueron los peores desde la Gran Depresión.

Con la nueva estrategia se logró la estabilidad de precios en forma indirecta, dado que se procuró modificar las expectativas de los agentes así como las tasas de interés y el tipo de cambio. Debido a que estos elementos afectan a la demanda agregada, el Banco de México pudo determinar el comportamiento futuro de la inflación (Díaz de León y Greenham, 2000). Para 
provocar cambios en las tasas de interés internas, el Banco de México decretó cambios en el saldo mensual acumulado que mantiene la banca comercial con dicho banco (el "corto") 5 . Según las autoridades monetarias, la inflación fue un problema ocasionado por el exceso de demanda 6 . Por lo tanto, los repuntes inesperados de la inflación real o las expectativas de inflación se manejaban mediante la reducción del "corto", induciendo de esta forma a los bancos comerciales a aumentar las tasas de interés.

Desde la adopción del régimen cambiario flotante, la evolución de la política monetaria ha incluido los siguientes elementos principales. Primero, la adopción de objetivos cuantitativos basados en agregados como la base monetaria, especialmente durante el período 1995-1997. Segundo, a principios de 1998, el Banco de México anunció un cambio en su estrategia y comenzó a adoptar metas inflacionarias. En particular, en el programa monetario de 1999 se estableció una meta inflacionaria anual máxima del 13\% para el Índice Nacional de Precios al Consumidor (INPC) y se propuso, como compromiso para los siguientes cinco años, una convergencia gradual con la tasa de inflación de los principales socios comerciales del país. En 2001, el

\footnotetext{
5 A partir del 21 de enero de 2008, el Banco de México adoptó como objetivo operacional la tasa de interés interbancaria a un día ("tasa de fondeo bancario") en sustitución del saldo sobre las cuentas corrientes que la banca mantiene en el propio banco central (objetivo conocido como el "corto").[Fuente: Banco de México, http://www. banxico.org.mx/politica-monetaria-e-inflacion/material-de-referencia/ intermedio/politica-monetaria/\%7B16DC84D2-A904-6373-28E329659E530FDB\%7D.pdf ]

${ }^{6}$ Garriga (2010) va más allá y afirma que las nuevas autoridades monetarias no creían en la existencia de una relación de correspondencia entre el desempleo y la inflación.
}

objetivo de la política monetaria era estabilizar el INPC en un 3\%. Sin embargo, a mediados de 2002, el Banco de México anunció que este compromiso tendría un margen de tolerancia de \pm 1 punto porcentual.

Castellanos (2000) señala que las modificaciones del "corto" también han tenido una gran repercusión en toda la estructura de la tasa de interés. Indica que el efecto es más fuerte para las tasas de interés a corto plazo. La magnitud de este efecto ha cambiado en el tiempo, y desde junio de 1998 ha sido más inestable. En realidad, Gaytán y González (2006) encontraron que se ha producido un cambio en los mecanismos de transmisión de la política monetaria como resultado de la política de metas inflacionarias. Estos autores sostienen que las fluctuaciones cambiarias estaban teniendo un efecto menor en la formación de los precios, la formación de las expectativas de inflación y las tasas de interés nominales, mientras que los cambios en las tasas de interés nominales han sido más efectivos para incidir en el tipo de cambio real y la inflación.

En resumen, en esta sección se ha descrito la política monetaria adoptada por el Banco de México para controlar la inflación. Los instrumentos utilizados han ido variando a lo largo del tiempo. Durante los primeros años, los controles cambiarios y de precios se emplearon intensamente. Más tarde, el régimen de tipo de cambio fijo fue reemplazado por una tasa flotante para que el Banco de México tuviera la libertad de controlar los agregados monetarios, las tasas de interés, o ambos. Durante esta segunda etapa, las tasas de interés fueron el principal instrumento utilizado para controlar la inflación. En la medida en que las tasas de interés así como los tipos de cambio afectan al producto y, en consecuencia, al empleo, es dable pensar que la política monetaria tiene el potencial de repercutir profundamente en el desempleo. 


\section{IV}

\section{El mercado laboral mexicano}

Esta sección tiene un doble propósito. En primer lugar, en ella se describen las características clave del mercado laboral de México con el objetivo de brindar una comprensión más acabada de la dinámica del empleo y del modo en que el desempleo puede responder a perturbaciones de política. En segundo término, a la luz de algunas críticas surgidas con respecto a la tasa de desempleo oficial, se calcula aquí una tasa de desempleo alternativa y se la utiliza para determinar el efecto de la política monetaria.

\section{La naturaleza del mercado laboral mexicano}

Existen dos posturas opuestas sobre la naturaleza del mercado laboral mexicano. Por una parte, está la idea de que es un mercado fuertemente regulado por leyes que impiden la creación de empleos (Heckman y Pagés, 2001; Gill, Montenegro y Dömeland, 2001). Según esta postura, el crecimiento del producto no se traduce en incremento del empleo, sino en cambios en el salario real. Algunos afirman que las disposiciones en materia de seguridad del empleo (que incluyen la indemnización por despido) aumentan los costos de despido para las empresas. Estos costos desalientan a las firmas a despedir trabajadores cuando se produce una perturbación negativa y reducen la creación de empleos en épocas de crecimiento. Heckman y Pagés (2001) concluyen que México presenta uno de los índices más altos de seguridad del empleo de América Latina, lo que implica que tiene uno de los mercados laborales más regulados de la región.

La rigidez del empleo también puede acrecentarse debido a la existencia de sindicatos de trabajadores. Maloney (2009), por ejemplo, sostiene que los sindicatos mexicanos valoran más el empleo que los salarios, de manera que las fluctuaciones del producto afectan más a los salarios que al empleo. En esto pueden incidir varios factores. En primer lugar, México no tiene seguro de desempleo; por consiguiente, los trabajadores valoran más la estabilidad laboral que los salarios. En segundo lugar, desde comienzos del siglo XX, los sindicatos más importantes han tenido una relación cercana y prolongada con el gobierno y han cooperado en la aplicación de políticas destinadas a reducir las presiones inflacionarias. En particular, desde fines de la década de 1980, los sindicatos se han conformado, en general, con un crecimiento del salario nominal inferior a la inflación. En tercer lugar, el crecimiento de los empleos fue lento en relación con el aumento de la población durante el período que va de fines de los años ochenta a principios de los años noventa. Estos elementos explican por qué las fluctuaciones del producto se correlacionan más con las fluctuaciones del salario real que con los cambios en el empleo. También pueden explicar la conclusión a que llega Alcaraz de que los salarios reales no dependen del desempleo en el sector formal (Alcaraz, 2009)

Por otra parte, se argumenta que si bien el mercado laboral de México se encuentra marcadamente regulado por leyes laborales, en la práctica, el cumplimiento de dichas leyes es muy pobre. Asimismo, una serie de esquemas adoptados desde fines de los años ochenta han incrementado la flexibilidad laboral (Marshall, 2004). Entre estos esquemas encontramos el uso cada vez más frecuente de contratos a corto plazo para evitar la inflexibilidad del empleo permanente. Esto es particulamente efectivo en la industria maquiladora y en el sector de servicios, los de más rápido crecimiento en la economía mexicana desde fines de la década de 1980.

Asimismo, Alcaraz, Chiquiar y Ramos-Francia (2008) afirman que desde el año 2000 el porcentaje de empleo en el sector de servicios se ha acrecentado a expensas del porcentaje correspondiente a la industria manufacturera. Según estos autores, esta recomposición del empleo ha implicado no solo un aumento de la participación del sector de servicios en el empleo, sino también un engrosamiento del sector informal. La tendencia ascendente del empleo en el sector informal se ha dado a pesar de la diferencia entre los salarios en el empleo formal e informal. También encontraron evidencia de que la tasa de transición entre el empleo formal y el informal es mayor que la existente entre los sectores manufacturero y de servicios. Estos autores señalan que la mayor movilidad entre los sectores formal e informal (y viceversa) indicaría la existencia de rigideces institucionales del mercado de trabajo en el sector formal de México.

La existencia de un amplio sector informal ${ }^{7} \mathrm{com}$ pensa de alguna manera las rigideces generadas por la normativa laboral aplicable al sector formal. La cuestión es si esta fuerza compensatoria es suficientemente

\footnotetext{
7 Alcaraz (2009) señala que este sector puede llegar a representar hasta el $40 \%$ de la fuerza de trabajo mexicana.
} 
acentuada para poder caracterizar al mercado de trabajo mexicano como bastante flexible.

En resumen, dentro del mercado laboral mexicano coexisten dos tipos de factores. Por una parte, existen algunas instituciones de dicho mercado - concretamente, la normativa laboral y los sindicatos- que aumentan su rigidez en el empleo al tiempo que incrementan la flexibilidad del salario real. Por otra, se encuentran elementos que compensan el efecto negativo de estas instituciones en la flexibilidad laboral, en especial la aplicación deficiente de la legislación del trabajo y la existencia del sector informal. A priori, no se sabe cuál de estos dos tipos de factores tiene un mayor efecto en la flexibilidad en el empleo.

\section{2. ¿Cuán precisas son las cifras de desempleo en México?}

Desde afuera, las bajas tasas oficiales de desempleo en México representan un enigma. ¿Cómo puede ser que un país con tasas de desempleo tan bajas crezca tan poco? En un nivel cercano al 3,5\%, la tasa de desempleo de México durante aproximadamente los últimos 20 años ha sido en promedio una de las más bajas entre los países miembros de la Organización de Cooperación y Desarrollo Económicos (OCDE), donde solo Luxemburgo y la República de Corea presentan tasas menores ${ }^{8}$. Sin embargo, la tasa media de crecimiento anual del PIB per cápita de México ha sido de 1,2\%, muy inferior al 5,8\% registrado en la República de Corea, un país con tasas de desempleo similares 9 .

Varios autores han sostenido que las bajas tasas de desempleo oficiales de México pueden deberse a la combinación de diversos elementos, a saber: i) la falta de beneficios de desempleo; ii) la existencia de un sector informal bastante bien remunerado y amplio; iii) las altas tasas de migración de mano de obra a los Estados Unidos de América; y iv) la exclusión de las áreas rurales en el cálculo de las tasas de desempleo, dado que este es mayor en esas áreas que en las urbanas.

\footnotetext{
${ }^{8}$ Entre 1987 y 2004 la tasa media de los países de la OCDE fue de un $5,93 \%$ (http://stats.oecd.org).

${ }^{9}$ Entre 1987 y 2004 el PIB real total de México aumentó a una tasa media anual del $3,01 \%$, mientras que su población creció a una tasa media de un $1,8 \%$ por año. En la República de Corea, en cambio, el producto se expandió a una tasa media anual del $6,2 \%$, mientras que el incremento poblacional fue de un $0,33 \%$ durante el mismo período (los datos sobre el producto se obtuvieron de la página web de la OCDE http://stats.oecd.org, mientras que aquellos sobre el crecimiento poblacional se obtuvieron de la publicación World Population Prospects: The 2006 revision, Naciones Unidas, 2007, cuadro A.8).
}

Sin embargo, si bien algunos de estos factores pueden explicar las bajas tasas de desempleo en México, una explicación más significativa se vincula al método de cálculo empleado. Fleck y Sorrentino (1994) y Martin (2000), por ejemplo, señalan que si se siguen los conceptos y la metodología utilizada por la Oficina de Estadísticas Laborales de los Estados Unidos de América, las tasas de desempleo de México aumentan entre un $40 \%$ y un 70\%. Revenga y Riboud (1993) llegan a la misma conclusión, aunque por una razón diferente: en las estadísticas oficiales no se toma en cuenta a los empleados temporales, o a aquellos que están desempleados pero no incluidos en las encuestas ${ }^{10}$.

Para resolver una de las principales críticas a las estadísticas oficiales, en este trabajo se recalculó la tasa de desempleo de México utilizando la Encuesta Nacional de Empleo Urbano (ENEU) trimestral y siguiendo los criterios de la Encuesta Continua de Población. Primero, se consideró únicamente a los trabajadores entre 16 años y 75 años ${ }^{11}$. En segundo término, para obtener la tasa de desempleo total, solo se consideraron las 16 ciudades originales incluidas en la ENEU. En tercer lugar, no se consideró como empleados a quienes estaban en huelga o habían sido despedidos debido a escasez de capital de trabajo o de materias primas, o por falta de reparaciones. También se excluyeron las personas que trabajaban sin remuneración hasta 15 horas semanales. Es decir, no fueron categorizados como parte de la población económicamente activa (PEA) o como empleados. La serie resultante permite comparar las tasas de desempleo de México directamente con las de los países de la ocDE.

En el gráfico 1 se muestra la evolución de las tasas de desempleo oficial y alternativa. En comparación con las tasas oficiales, la medida alternativa es casi $100 \%$ más alta, es decir, la tasa media de desempleo correspondiente al período 1987-2004 se acrecienta del 3,4\% al $6,4 \%$. No obstante, el comportamiento general de las dos series es bastante similar. La principal diferencia se observa durante el período anterior a la crisis de 1995. A principios de la década de 1990, ambas series muestran una ligera tendencia ascendente que alcanza su culminación a fines de 1995. La tasa de desempleo comienza a descender rápidamente en 1996 y alcanza el nivel más

\footnotetext{
${ }^{10}$ Esto es particularmente importante cuando una persona está desempleada y no está buscando trabajo en el momento de una encuesta, pero encuentra trabajo antes de que se lleve a cabo la siguiente encuesta; por lo tanto, figura como empleado.

${ }^{11}$ Con anterioridad a 2005, en las estadísticas mexicanas se incluía a los trabajadores a partir de los 12 años. En respuesta a las críticas, en el año 2005 se elevó el límite de edad mínimo a 14 años.
} 
GRÁFICO 1

\section{México: tasas de desempleo oficial y alternativa}

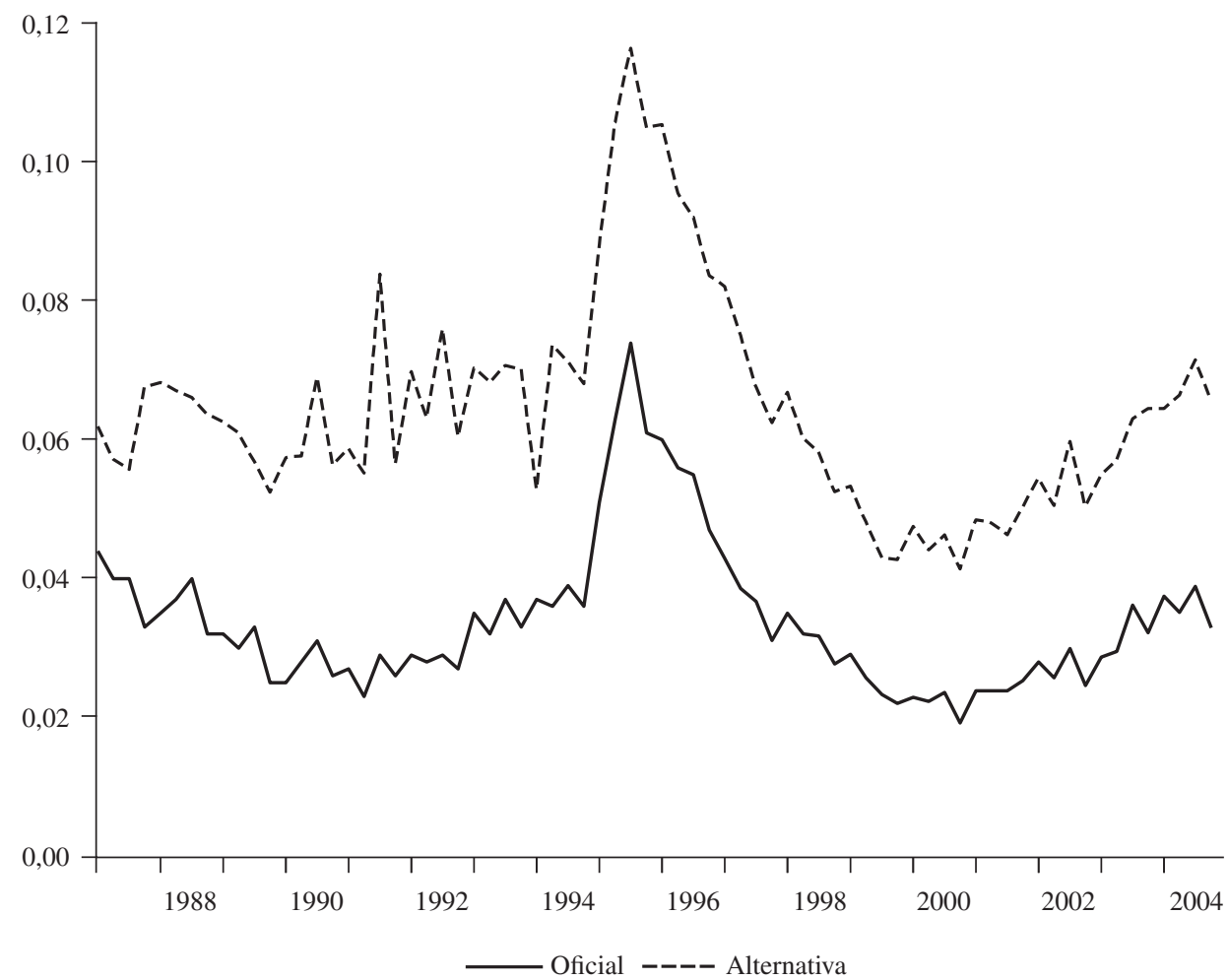

Fuente: Instituto Nacional de Estadística y Geografía (INEGI), Encuesta Nacional de Empleo Urbano (ENEU), varios años.

bajo a fines de 2000 en ambas series. Sin embargo, esta mejora es de poca duración, dado que al año siguiente el desempleo retoma la tendencia ascendente. Ambas series de desempleo registran grandes fluctuaciones cíclicas así como variaciones de corto plazo.

En la sección anterior se describieron las principales políticas monetarias y fiscales adoptadas durante el período 1987-2003. Se señaló que el objetivo primordial de ambos tipos de políticas era controlar la inflación. Para tal fin, no obstante, el Banco de México necesitó frenar la demanda agregada, lo que a su vez generó una contracción del producto y, como consecuencia, un incremento del desempleo. A continuación, se presenta la técnica econométrica utilizada en el análisis. 


\section{V}

\section{Metodología}

Se emplea un modelo de vectores autorregresivos estructurales (SVAR) para analizar el efecto dinámico de la política monetaria en el desempleo. Cabe mencionar que otra manera de efectuar el análisis es mediante el uso de un modelo de Markov-Switching de vectores autorregresivos. Sin embargo, debido al tamaño reducido de la muestra de que se dispone en este trabajo, los grados de libertad se habrían perdido rápidamente, razón por la que no se efectuó un análisis con dicho modelo. El presente análisis se complementa con la estimación de la función de respuesta al impulso y la descomposición de la varianza.

Por lo tanto,

$$
A X_{t}=B(L) X_{t-1}+\varepsilon_{t}
$$

donde $X_{t}$ es un vector de variables endógenas $(n x l)$; $A$ es una matriz de coeficientes $(n x n)$ que describe el efecto contemporáneo entre las variables; $B(L)$ es un polinomio de matrices ( $n x n)$ en el operador de retardos $L ;$ y $\varepsilon_{t}$ es un vector de innovaciones que en este modelo estructural se denominan shocks estructurales $(n x l)$. De este modo, cada una de las variables del sistema puede verse afectada por sus propios shocks idiosincrásicos y por los de las otras variables. Las matrices $A$ y $B(L)$ determinan cómo se transmiten las perturbaciones que afectan a cada variable a través del sistema, tanto contemporáneamente como en períodos subsiguientes. La forma reducida-autorregresiva puede expresarse de la siguiente manera:

$$
X_{t}=A^{-1} B(L) X_{t-1}+A^{-1} \varepsilon_{t}=C(L) X_{t-1}+e_{t}
$$

donde $e_{t}=A^{-1} \varepsilon_{t}$ describe la relación entre las innovaciones del modelo en su forma reducida y las innovaciones en su forma estructural. En realidad, las innovaciones del modelo en su forma reducida son combinaciones lineales de los shocks estructurales.

Una condición necesaria para establecer las relaciones entre las innovaciones del modelo en su forma reducida y los shocks estructurales, es que el modelo sea identificable. Como es bien sabido, para que el modelo esté exactamente identificado se requiere imponer $\frac{n^{2}-n}{2}$ restricciones entre las relaciones de las innovaciones del modelo en su forma reducida y los shocks estructurales.

En la literatura sobre vectores autorregresivos estructurales se pueden encontrar diferentes métodos de identificación. Blanchard y Diamond (1989) utilizan supuestos a priori sobre los signos de los parámetros estructurales para identificar el rango de valores de la matriz $A$ que concuerda con los datos. Shapiro y Watson (1988) y Blanchard y Quah (1989) emplean supuestos sobre multiplicadores de largo plazo para realizar la identificación. Blanchard y Quah (1989) comienzan con choques ortogonales. En un modelo bivariado, se supone que una de estas innovaciones representa un shock de oferta y otra innovación un shock de demanda. Por ejemplo, para diferenciar los shocks de productividad de los de demanda se supone que los segundos no afectan al producto real en el largo plazo, mientras que los primeros sí lo hacen. Por otra parte, Sims (1980) sostiene que para realizar la identificación de los shocks se deben emplear restricciones contemporáneas, esto es, innovaciones de política monetaria que se identifican con frecuencia mediante el supuesto de que los cambios en la tasa de interés no afectan a la inflación en el mismo período, porque los precios son rígidos y responden con un rezago. En el presente análisis, se sigue el enfoque de Sims (1980) para identificar el modelo.

\section{Definición de variables y datos}

En el modelo SVAR aquí utilizado se incluyen las siguientes variables: las tasas de desempleo, la brecha del producto (dado que la demanda de mano de obra es una demanda derivada que depende de las fluctuaciones del producto) y un indicador de la política monetaria. También se incluyen tres variables que se estima afectan al desempleo mexicano: el producto de los Estados Unidos de América, un indicador de la política fiscal y la productividad de la mano de obra. A continuación, se explica la inclusión de estas variables en el presente análisis.

Durante el período de análisis de este estudio, se transitó de un régimen de tipo de cambio fijo a uno de tipo de cambio flotante. Bajo un régimen de tipo de cambio fijo, los bancos centrales pierden independencia en la determinación de su política monetaria, mientras que 
con uno de cambio flotante tienen el control de la política monetaria y pueden utilizarlo para lograr metas específicas. Además, con un régimen de tipo de cambio flotante las tasas de interés y las de tipo de cambio se vuelven interdependientes. Los efectos que tienen en el producto y, por lo tanto, en el desempleo operan a través de distintos mecanismos. En lugar de usar el tipo de cambio y las tasas de interés en forma independiente como instrumentos de política monetaria, aquí se utiliza un índice compuesto de política monetaria. Específicamente, se emplea el índice de condiciones monetarias (ICM), que captura el efecto total (directo e indirecto) del tipo de cambio y la tasa de interés en el producto interno.

El ICM se mide comúnmente como un promedio ponderado de las tasas de interés de corto plazo y el tipo de cambio, tomando como referencia un período base. Los pesos o ponderaciones se definen por el efecto relativo del tipo de cambio y las tasas de interés en la inflación y pueden ser determinados de manera empírica $^{12}$. De este modo, el ICM determina el grado de estrechamiento o relajamiento de las condiciones monetarias de una economía al recoger las desviaciones de estas dos variables con respecto a un período base. Así, menores tasas de interés impulsan condiciones monetarias más relajadas o expansivas y mayores tasas denotan contracciones más restrictivas, a lo que también se puede llegar mediante una apreciación de la moneda nacional, o una combinación de ambas medidas.

En el presente trabajo se utiliza el ICM como un indicador de la orientación de la política monetaria. Por ejemplo, dicho indicador calculado en relación con un período base señala si la política se ha vuelto "más restrictiva" o "más laxa" con respecto a ese período. En esta construcción, el valor absoluto del ICM no proporciona la base para una interpretación, sino más bien revela la orientación de la política monetaria. Al utilizar los coeficientes estimados de la tasa de interés y tipo de cambio en el vector de cointegración, la razón o el peso del ICM resulta ser 1 a 4.2, lo que indica que el tipo de

\footnotetext{
12 Los pesos relativos de la tasa de interés y el tipo de cambio para el ICM se obtuvieron a través de la ecuación de la demanda agregada. En la literatura se han sugerido tres enfoques para el cálculo de los pesos relativos: i) el enfoque de una sola ecuación, que consiste en el cálculo de una ecuación del producto o de los precios; ii) el enfoque de la proporción del comercio exterior, que radica en el cálculo de una ecuación de la relación de las exportaciones a largo plazo respecto del PIB; iii) el enfoque de ecuaciones múltiples, que estriba en el cálculo del sistema de ecuaciones mediante la cointegración de las variables. Se utilizan los modelos de vectores autorregresivos y la prueba de cointegración de Johansen para evitar el sesgo por variables omitidas, exogeneidad dinámica y problemas de retroalimentación. El enfoque de cointegración resuelve estos problemas.
}

cambio tiene mayor repercusión en el producto agregado que los efectos de la tasa de interés ${ }^{13}$.

La brecha de producto se define como la diferencia entre el producto observado y el potencial, y se parte del supuesto de que el producto potencial puede caracterizarse como generado por un proceso estocástico y puede obtenerse mediante el filtro de Hodrick-Prescott ${ }^{14}$. De ese modo, la brecha de producto es $\tilde{y}=\left(y-y^{*}\right)$, donde $y^{*}$ es el componente permanente del producto, mientras que $y$ es el PIB observado.

Dado que en el programa de estabilización de la economía mexicana se incluyó la reducción de los déficits fiscales, es necesario tomar en cuenta el efecto de la política fiscal en el desempleo. Por lo tanto, en el modelo empleado en este trabajo se incluye el superávit primario estructural, que se define como la diferencia entre el déficit observado y el cíclico. Esto es, el déficit estructural es el déficit del gobierno ajustado por los ciclos económicos medidos por la razón entre el producto potencial y el observado. De acuerdo con Pastor y Villagómez (2007), se consideró el déficit del gobierno ajustado por el ciclo económico ${ }^{15}$. Este ajuste se efectúa multiplicando los ingresos fiscales por la razón entre el PIB potencial y el PIB observado, elevado a la elasticidad ingreso de ese componente. Para construir el superávit primario estructural se utilizaron datos presupuestarios de la Secretaría de Hacienda y Crédito Público.

En varios estudios se ha demostrado la cointegración de los ciclos económicos de México y de los Estados Unidos de América. Este hallazgo se explica debido a que más del $90 \%$ de las exportaciones mexicanas se destinan

\footnotetext{
${ }^{13}$ En estudios empíricos recientes se ha demostrado que desde 2001 se ha producido un cambio en el mecanismo de transmisión de la política monetaria, como consecuencia de una nueva estrategia antiinflacionaria basada en la política de metas de inflación. Gaytán y González (2006) señalan que el producto y la inflación se han vuelto más sensibles a las tasas de interés, mientras que el tipo de cambio real ha perdido importancia. Los resultados obtenidos en este trabajo difieren un poco, dado que en este caso el período de análisis se remonta a 1987, cuando el tipo de cambio era el ancla nominal. De hecho, el tipo de cambio nominal fue el principal instrumento hasta 1994. ${ }^{14}$ La elección de una $\lambda$ grande en el filtro de Hodrick-Prescott implica la postura de que los choques de oferta son deterministas y que las variaciones en el producto se deben casi totalmente a los de demanda. La elección de una $\lambda$ muy pequeña involucra la postura de que la mayoría de las variaciones en el producto son asimismo variaciones en el producto potencial o tendencial y, que por lo tanto, son generadas por choques de oferta.

15 De acuerdo con Hayford (2005), la lógica detrás de las medidas estructurales es que uno de los problemas para identificar los efectos de la política fiscal obedece a que los ingresos y los pagos de transferencias del gobierno responden a los ciclos económicos. Una forma de solucionar este problema es controlar el efecto del ciclo económico usando medidas estructurales de la política fiscal.
} 
a la economía estadounidense. A fin de tomar en cuenta la repercusión de este hecho en el desempleo en México, en el presente modelo se incluye la brecha de producto de los Estados Unidos de América, $\tilde{q}$, como indicador de la demanda externa. Por lo tanto, $\tilde{q}=\left(q-q^{*}\right)$, donde $q^{*}$ es el producto potencial estadounidense, calculado mediante el filtro de Hodrick-Prescott, y $q$ es el producto observado de los Estados Unidos de América.

Por último, también se incluye una proxy para el cambio tecnológico. Se ha sostenido que el cambio de tecnología que supone un ahorro de mano de obra puede afectar a las tasas de desempleo y, con el objeto de tomar en cuenta dichos cambios en la eficiencia tecnológica, se incluye la productividad de la mano de obra en el modelo como variable exógena. Dicha productividad se mide en términos de producción por trabajador empleado.

A fin de realizar el análisis empírico, se utilizan datos trimestrales disponibles desde el primer trimestre de 1987 hasta el cuarto trimestre de 2004, para un total de 74 observaciones. La brecha de producto se calcula a partir del PIB real, obtenido del Instituto Nacional de Estadística y Geografía (INEGI), mientras que la brecha de producto estadounidense se calcula a partir del PIB real de los Estados Unidos de América, de acuerdo con los principales indicadores económicos de la ocDE. El indicador de la política fiscal de México es el superávit fiscal, mientras que el indicador de la productividad de la mano de obra es la producción por trabajador.

Se utilizan tanto las series de desempleo oficial como la alternativa, ambas con ajuste estacional mediante el programa TRAMO-SEATs (por sus siglas en inglés). De igual modo, la brecha de producto mexicano y la estadounidense se obtuvieron empleando el filtro de Hodrick-Prescott con un valor $\lambda=1600$ después del ajuste estacional mediante el programa TRAMO-SEATS.

\section{Identificación}

El modelo SVAR incluye tres variables endógenas: la política monetaria (ICM), la brecha de producto $\left(y^{*}\right)$ y el desempleo $(u)$, y tres variables exógenas: la brecha de producto de los Estados Unidos de América $(\tilde{q})$, la productividad laboral $(p)$ y la política fiscal $(g)$. Se evaluó la exogeneidad de estas últimas tres variables de acuerdo con Hyeon-Seung (2005). El resultado fue $\chi^{2}=37,00716$.

16 La estimación de este estadístico se obtuvo para 48 grados de libertad y a un $87 \%$ de significación estadística.
El orden de la matriz $A$ es el siguiente. La política monetaria se muestra primero, seguida del exceso de demanda interna, y luego la tasa de desempleo. La política monetaria se identifica asumiendo que los cambios en la tasa de interés no afectan al exceso de demanda en el mismo período, porque los precios son rígidos y responden con un rezago. Esto implica que $\alpha_{21}=0$. Dada la existencia de un gran sector informal y el alto grado de transición entre el empleo formal y el informal (véase la sección IV, subsección 1), las tasas de desempleo no cambiarán necesariamente a medida que cambie el producto. Las fluctuaciones del producto generarán flujos entre los sectores formal e informal, de modo que la tasa de desempleo se mantendrá bastante rígida. Esto se traduce en $\alpha_{32}=0$. Es importante destacar que la rigidez del mercado de trabajo no se debe a una legislación laboral estricta que impide que las fluctuaciones en el producto afecten al desempleo, sino que la rigidez obedece a la existencia de un amplio sector informal y una alta movilidad laboral entre los sectores formal e informal, de manera que el desempleo no se ve afectado contemporáneamente por las fluctuaciones en el producto. Resulta paradójico que en México la rigidez del mercado laboral sea el resultado de la existencia de un mercado de trabajo que es en efecto muy flexible ${ }^{17}$.

La tercera restricción se deriva del hecho de que la política monetaria no depende del desempleo, es $\operatorname{decir} \alpha_{12}=0$.

\footnotetext{
17 Calderon-Madrid (2000); Alcaraz, Chiquiar y Ramos-Francia (2008) y Alcaraz (2009) presentan alguna evidencia del alto grado de movilidad laboral entre los mercados formal e informal. La existencia de un amplio mercado de trabajo informal estrechamente entrelazado con el formal significa que las fluctuaciones en el producto no necesariamente se traducen en fluctuaciones del desempleo, o viceversa. En otras palabras, la existencia de un gran mercado laboral informal indica que la relación entre los componentes cíclicos del producto y el desempleo no es necesariamente lineal o contemporánea. Por el contrario, se puede observar que un cambio determinado en el producto provocará una mayor movilidad laboral entre los sectores formal e informal, de manera que la tasa de desempleo se mantiene constante. Si se considera, por ejemplo, que el empleo se descompone en sus componentes formal $\left(e_{f}\right)$ e informal $\left(e_{i}\right)$, lo siguiente debería ser cierto: $u=1-e_{f}-e_{i}$. Es decir, se van a producir variaciones en el desempleo solo si el empleo formal y el informal se mueven en la misma dirección, lo que en la realidad no sucede. Por lo tanto, el efecto de las variaciones del producto en el desempleo será bastante leve, salvo que el sector informal no tenga la flexibilidad necesaria. Esto es cierto incluso frente a las rigideces institucionales que mencionan Heckman y Pagés (2001).
} 


\section{VI}

\section{Análisis empírico}

En esta sección se presenta la estimación de los parámetros y se llevan a cabo algunos análisis complementarios, tales como el análisis de respuesta al impulso y el de descomposición de la varianza. También se realizan pruebas para comprobar la robustez de los resultados.

\section{Estimación del modelo SVAR}

Debido a que el interés principal en el modelo SVAR es poder determinar la relación entre las variables y no la estimación de los parámetros en sí mismos, se sigue la recomendación de Sims (1980) y Sims, Stock y Watson (1990) en contra de la diferenciación, incluso si las variables contienen una raíz unitaria. Sims, Stock y Watson (1990) sostienen que transformar el modelo a su forma estacionaria es en muchos casos innecesario, debido a que en modelos en que se combinan variables no estacionarias con variables estacionarias, los estimadores de los parámetros del modelo tendrán distribuciones estándares. No obstante, en este trabajo se han agregado algunos componentes deterministas, de modo tal que se obtiene un VAR estacionario.

Por lo tanto, puesto que ambas series de desempleo (la oficial y la alternativa) no son estacionarias a lo largo del período de análisis ${ }^{18}$, y dado que se quiere determinar la persistencia de los choques de política monetaria en lugar de asumir que los efectos son permanentes, se agrega al modelo una tendencia determinista para obtener un VAR estacionario, así como una tasa de desempleo estacionaria. En la medida en que la política monetaria se puede ver afectada por las expectativas de inflación, dicha variable se agrega al modelo. Se utilizó la inflación rezagada como una proxy de la inflación esperada. Si bien la inflación rezagada fue estadísticamente significativa, solo en la ecuación del ICM la inclusión de esta variable no tuvo un efecto cualitativo en el resto de los resultados. Los resultados del análisis incorporando las

\footnotetext{
18 Véase el cuadro A.2 del apéndice, donde se incluyen las pruebas de raíz unitaria. Se utiliza la prueba de Lee y Strazicich (2003) de raíz unitaria para dos quiebres estructurales basada en los multiplicadores de Lagrange.
}

expectativas de inflación se muestran en el apéndice (véase el cuadro A.1). Por lo tanto, el presente análisis se basa en un modelo parsimonioso que no incluye la inflación esperada.

Antes de estimar el modelo SVAR, se evalúan las restricciones de identificación $\left(\alpha_{21}=\alpha_{32}=\alpha_{13}=0\right)$. La razón de verosimilitudes fue 0,003 y no resultó significativa al compararla con los valores críticos de la distribución chi-cuadrada con tres grados de libertad; por lo mismo, la hipótesis conjunta no pudo ser rechazada. El orden del modelo se determinó utilizando los criterios de información de Akaike y el criterio bayesiano de información, lo que dio como resultado dos rezagos. Cuando se utilizó la tasa de desempleo oficial, los coeficientes del modelo SVAR estimados no resultaron ser estadísticamente significativos o tenían los signos incorrectos. De hecho, el impacto inicial de una perturbación en la política monetaria fue una reducción de la tasa de desempleo, resultado claramente contrario a lo que la teoría económica indica al respecto ${ }^{19}$.

En el cuadro 1 se enumeran los coeficientes del modelo SVAR estimados utilizando la serie de desempleo alternativa. Como se puede ver, no hay evidencia de errores de especificación en ninguna de las ecuaciones. En el cuadro 1 se agrupa la suma de los coeficientes sobre los dos rezagos de cada variable y la prueba de Wald para comprobar su significancia conjunta; los valores $p$ se reportan entre paréntesis. Los resultados son los esperados: una política monetaria restrictiva aumenta el desempleo y reduce la demanda interna; una demanda interna más elevada disminuye el desempleo. El superávit fiscal reduce la demanda interna, mientras que el efecto en el desempleo tiene el signo correcto, pero no es significativo. Los shocks al producto estadounidense aminoran el desempleo e incrementan la demanda interna, al tiempo que los de productividad aumentan la demanda interna.

\footnotetext{
${ }^{19}$ En el gráfico 3 se comparan los resultados de las funciones impulsorespuesta correspondientes a las dos tasas de desempleo (modelo 1 y modelo 5).
} 
CUADRO 1

Modelo SVAR estimado

(valores p entre paréntesis)

\begin{tabular}{|c|c|c|c|}
\hline & $\begin{array}{l}\text { Desempleo } \\
\quad \operatorname{Ln}(u)\end{array}$ & $\begin{array}{c}\text { Demanda interna } \\
\left(y-y^{*}\right)\end{array}$ & $\begin{array}{l}\text { Política monetaria } \\
\text { (ICM) }\end{array}$ \\
\hline Logaritmo natural Ln(u) & $\begin{array}{c}0,759 \\
(0,000)\end{array}$ & $\begin{array}{c}-0,351 \\
(0,081)\end{array}$ & $\begin{array}{c}0,516 \\
(0,398)\end{array}$ \\
\hline$\left(y-y^{*}\right)$ & $\begin{array}{l}-0,061 \\
(0,090)\end{array}$ & $\begin{array}{c}0,568 \\
(0,000)\end{array}$ & $\begin{array}{c}0,351 \\
(0,263)\end{array}$ \\
\hline$L(I C M)$ & $\begin{array}{c}0,024 \\
(0,064)\end{array}$ & $\begin{array}{l}-0,067 \\
(0,007)\end{array}$ & $\begin{array}{c}0,582 \\
(0,000)\end{array}$ \\
\hline Producto de los Estados Unidos & $\begin{array}{l}-0,183 \\
(0,014)\end{array}$ & $\begin{array}{c}0,293 \\
(0,036)\end{array}$ & $\begin{array}{c}0,315 \\
(0,457)\end{array}$ \\
\hline$\left(q-q^{*}\right)$ & $\begin{array}{l}-0,017 \\
(0,943)\end{array}$ & $\begin{array}{c}1,809 \\
(0,000)\end{array}$ & $\begin{array}{c}5,175 \\
(0,000)\end{array}$ \\
\hline Productividad $(t)$ & $\begin{array}{c}0,153 \\
(0,758)\end{array}$ & $\begin{array}{l}-2,711 \\
(0,005)\end{array}$ & $\begin{array}{l}-7,690 \\
(0,009)\end{array}$ \\
\hline Superávit fiscal $(g)$ & $\begin{array}{c}0,0008 \\
(0,884)\end{array}$ & $\begin{array}{l}-0,038 \\
(0,001)\end{array}$ & $\begin{array}{l}-0,011 \\
(0,744)\end{array}$ \\
\hline Tendencia temporal & $\begin{array}{c}1,766 \\
(0,012)\end{array}$ & $\begin{array}{l}-3,138 \\
(0,018)\end{array}$ & $\begin{array}{l}35,167 \\
(0,000)\end{array}$ \\
\hline Variable ficticia 95 & 0,834 & 0,811 & 0,837 \\
\hline$R^{2}$ ajustado & & & \\
\hline
\end{tabular}

Logaritmo de la función de verosimilitud-339,93

Portmanteau (12) 105,1633 (0,1311)

Fuente: cálculos de los autores.

Nota: el cuadro contiene la suma de los coeficientes sobre los dos rezagos de cada variable; asimismo, la prueba de Wald para la significancia conjunta de ambos rezagos de cada variable. Se presenta la prueba de Portmanteau conjunta para residuos de ruido blanco.

ICM: índice de condiciones monetarias.

\section{Funciones de respuesta al impulso}

Ya que estamos particularmente interesados en los efectos de las innovaciones de política monetaria en la tasa de desempleo, en el gráfico 2.A se muestra la dinámica del desempleo a un ajuste exógeno de una política monetaria restrictiva, junto con sus bandas de confianza. Los resultados permiten ver que el desempleo responde en la misma dirección a shocks positivos de política monetaria, lo que indica que un ajuste exógeno de una política monetaria restrictiva del $1 \%$ genera un aumento del desempleo de 0.26 puntos porcentuales luego de dos trimestres. Después de cinco años, el desempleo sigue siendo 0.06 puntos porcentuales más alto de lo que habría sido sin el shock de política monetaria restrictiva. Dado que este es un VAR estacionario, todas las innovaciones tienen un efecto temporal, pero el efecto de la política monetaria es persistente, de manera que el shock solo se desvanece gradualmente. En respuesta a una política monetaria restrictiva, el desempleo se incrementa siguiendo un patrón de U invertida, como el que se aprecia en otros estudios ${ }^{20}$. Se puede observar que después de tres trimestres, el desempleo llega a su punto máximo antes de retornar lentamente a su nivel original.

Los resultados de este estudio indican que el desempleo en el mercado laboral mexicano se ajusta más rápidamente que el desempleo en Suecia, donde Alexius y Holmlund (2007) estimaron que ante un shock de política monetaria restrictiva, el desempleo alcanza su efecto máximo luego de nueve trimestres. En un estudio en que se evalúa el impacto de un shock de política monetaria restrictiva en el desempleo de los

\footnotetext{
20 Véanse, por ejemplo, Alexius y Holmlund (2008) y Ravn y
} Simonelli (2008). 


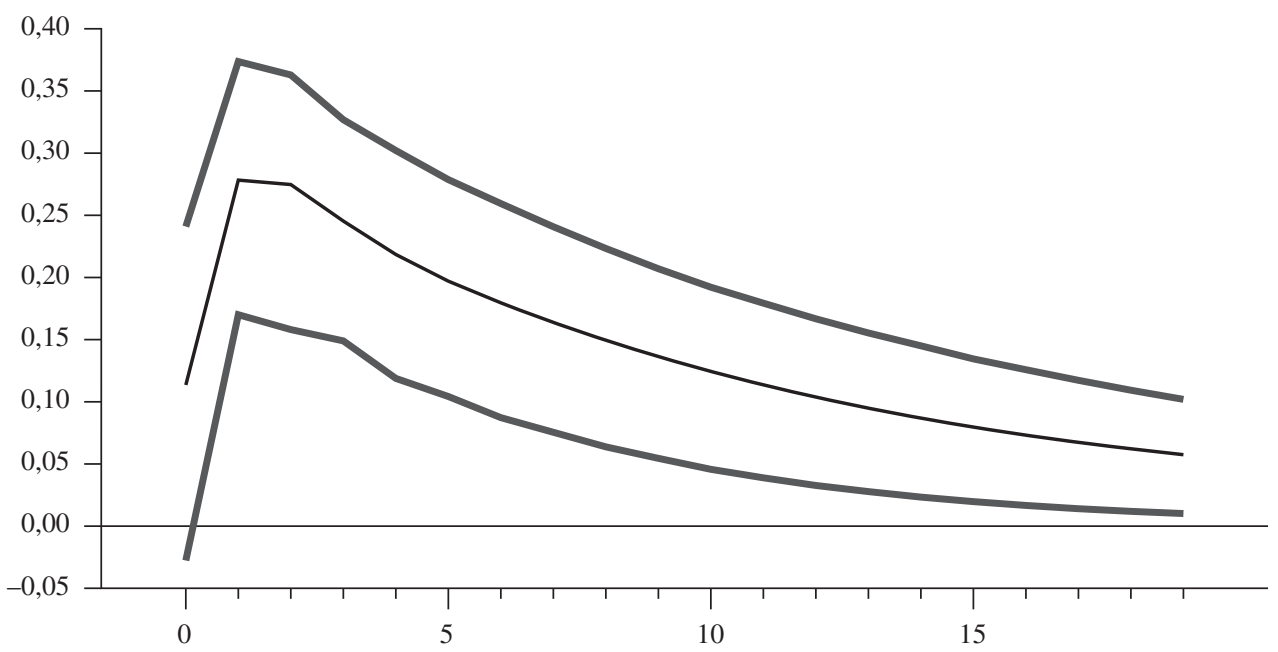

Fuente: cálculos de los autores.

Nota: respuesta a un shock de una desviación estándar en la política monetaria. Los intervalos de confianza se calcularon mediante el método de Monte Carlo. De acuerdo con las recomendaciones de Sims y Zha (1999), se reportan bandas de percentiles.

Estados Unidos de América, Ravn y Simonelli (2008) concluyeron que la mitad del efecto máximo de ese shock se desvanece luego de 10 trimestres y desaparece por completo después de 10 años.

Por lo tanto, el efecto de una política monetaria restrictiva en el desempleo es menos persistente en México que en Suecia, y tiene un comportamiento similar al efecto que causa en el desempleo de los Estados Unidos de América. La velocidad del ajuste se puede atribuir a la existencia de un amplio sector informal y a una serie de esquemas que han aportado algo de flexibilidad, a pesar de la fuerte regulación en el mercado de trabajo formal mexicano. La normativa laboral y los sindicatos de trabajadores, por otra parte, podrían explicar la baja elasticidad del empleo ante shocks de políticas monetarias restrictivas. Los resultados de esta investigación son consistentes con los reportados anteriormente en algunos estudios para América Latina ${ }^{21}$. Sin embargo, a diferencia de esos

21 Véanse, por ejemplo, Loboguerrero y Panizza (2005) y González Anaya (2002). trabajos, en el presente estudio se sostiene que la existencia de un amplio sector informal y un creciente sector de servicios podrían explicar la velocidad de ajuste encontrada.

En el gráfico 2B se observa que un shock de política monetaria restrictiva tiene un efecto negativo en el exceso de demanda interna. El efecto alcanza su punto máximo luego del primer trimestre, en comparación con el segundo trimestre en el caso del desempleo. La mitad del efecto máximo desaparece después del séptimo trimestre. Estos resultados son estadísticamente significativos y diferentes de cero. Como en el caso del desempleo, la función de respuesta al impulso del exceso de demanda interna vuelve a cero luego de seis años. Este resultado indica que el producto cae cuando la política monetaria es restrictiva, lo que es lógico, puesto que las tasas de interés real afectan al costo de capital y, por lo tanto, al consumo y la inversión.

En resumen, los resultados indican que un ajuste exógeno de la política monetaria restrictiva aumentará el desempleo al tiempo que hará descender el producto por debajo de su nivel potencial. 


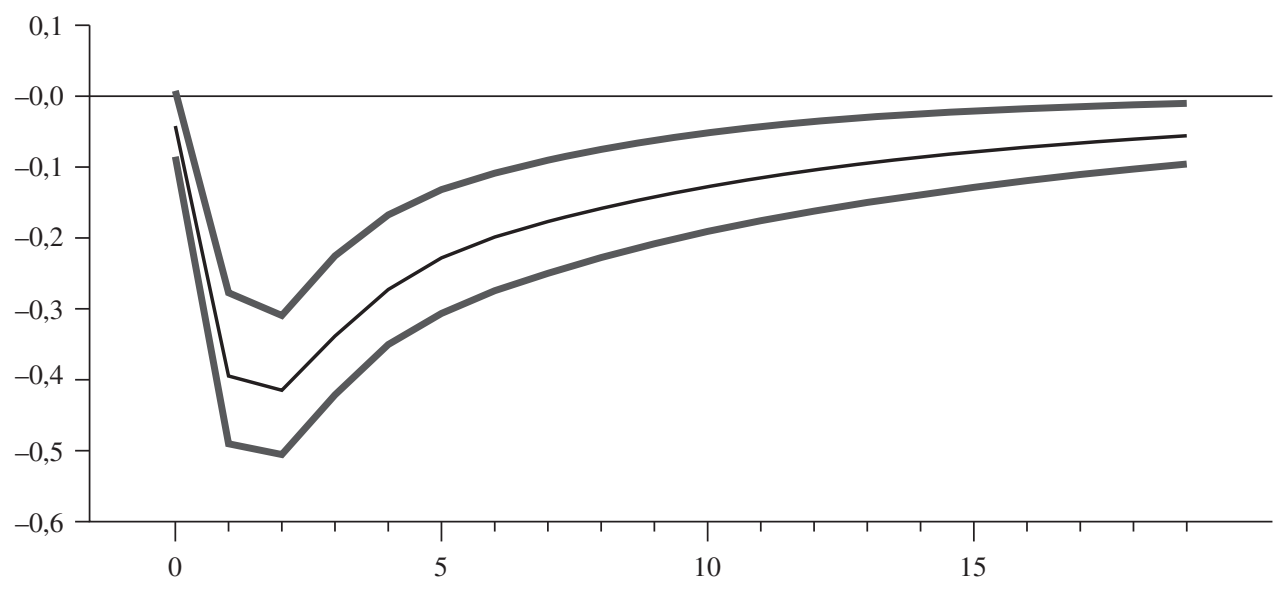

Fuente: cálculos de los autores.

Nota: respuesta a un shock de una desviación estándar en la política monetaria. Los intervalos de confianza se calcularon mediante el método de Monte Carlo. De acuerdo con la recomendación de Sims y Zha (1999), se reportan bandas de percentiles.

Descomposición de la varianza

\begin{tabular}{|c|c|c|c|c|c|c|c|c|c|c|c|c|}
\hline & \multicolumn{4}{|c|}{$\operatorname{Ln}(u)$} & \multicolumn{4}{|c|}{$\tilde{y}$} & \multicolumn{4}{|c|}{$I C M$} \\
\hline & 1 & 10 & 20 & 30 & 1 & 10 & 20 & 30 & 1 & 10 & 20 & 30 \\
\hline$\underset{\sim}{\operatorname{Ln}}(u)$ & 93,35 & 71,71 & 69,71 & 69,50 & 5,47 & 4,28 & 4,40 & 4,42 & 1,17 & 23,99 & 25,80 & 26,07 \\
\hline$\tilde{y}$ & 0,00 & 9,07 & 11,17 & 11,40 & 100 & 59,34 & 56,08 & 55,73 & 0,00 & 31,57 & 32,71 & 32,85 \\
\hline ICM & 0,00 & 5,02 & 6,92 & 6,88 & 0,72 & 4,94 & 5,04 & 5,04 & 99,27 & 90,02 & 88,26 & 88,06 \\
\hline
\end{tabular}

Fuente: cálculos de los autores.

Nota: las cifras de la segunda fila son horizontes (es decir, trimestres); las demás cifras son estimaciones que fueron redondeadas en dos decimales; debido a ello los errores de redondeo pueden en algunos casos impedir una descomposición porcentual perfecta.

ICM: índice de condiciones monetarias.

$\mathrm{Ln}$ : logaritmo natural.

$\tilde{y}$ : brecha de producto.

\section{Descomposición de la varianza}

A continuación se examinará la varianza del error de predicción. La descomposición de la varianza del error de predicción informa, en distintos horizontes del tiempo, el porcentaje de volatilidad que registra cada variable debido a los shocks de las demás. Es decir, indica la proporción del efecto que, en forma dinámica, tienen todas las variables en el resto. En el cuadro 2 se muestra la descomposición de la varianza del error de predicción en los horizontes de 1, 10, 20 y 30 trimestres. Resulta interesante determinar qué porcentaje de las fluctuaciones en el desempleo se deben a shocks de política monetaria y a shocks de la demanda interna (primera fila). Los resultados indican que la varianza del desempleo es explicada por la política monetaria en un rango del $1 \%$ al $26 \%$, dependiendo del horizonte del tiempo, mientras que el exceso de demanda interna explica apenas el 5\% en el corto plazo y la proporción disminuye en el largo plazo al $4 \%$. Se puede observar que, en el corto plazo, la varianza del desempleo se explica por sí misma en un nivel cercano al 93\%, proporción que cae al $69 \%$ en un horizonte más largo. 


\section{Robustez}

Dado que la estimación del modelo SVAR se llevó a cabo sobre la base de una serie de supuestos específicos, se investigó la robustez de los resultados ante distintos conjuntos de supuestos. Se estimaron cuatro modelos adicionales al modelo base (véase el gráfico 3). En el modelo 2, por ejemplo, se incluye — como indicador de política monetaria - un ICM donde el peso o ponderador colocado sobre el tipo de cambio está en función del peso de las exportaciones de largo plazo con relación al PIB. El peso de las tasas de interés se normaliza respecto del tipo de cambio y de esa manera ambos suman la unidad. El modelo 3 es un modelo svar con tres rezagos debido a que, según el criterio de información de Akaike y el criterio bayesiano de información, existen argumentos en favor de dos o tres rezagos. En el presente modelo base se optó por la especificación más parsimoniosa, es decir, dos rezagos.

En el modelo 4 se eliminó el supuesto de que el desempleo no se ve afectado por el producto y se dejó que el coeficiente fuera estimado por el modelo, tomando como supuesto, en cambio, que la política monetaria no responde contemporáneamente al exceso de demanda $\left(\alpha_{12}=0\right)$. Se mantiene el supuesto de que los shocks monetarios no afectan al exceso de demanda contemporáneamente $\left(\alpha_{21}=0\right)$ y que el desempleo no afecta a la política monetaria contemporáneamente $\left(\alpha_{13}=0\right)$. Los modelos 1 a 4 se estiman empleando la tasa de desempleo alternativa. El modelo 5, en cambio, es el modelo base en el que se emplea la tasa oficial de desempleo abierto.

En el gráfico 3 se muestra la función de respuesta al impulso del desempleo ante choques de política monetaria restrictiva correspondientes a los cinco modelos. A excepción del modelo 5, los resultados son bastante robustos, donde los efectos del shock alcanzan su nivel máximo luego de dos trimestres. La magnitud del efecto máximo varía entre 0.26 y 0.50 puntos porcentuales en respuesta a un shock de política monetaria restrictiva. Como ya se mencionó, los parámetros estimados mediante la tasa de desempleo oficial no son congruentes con la teoría económica.

Otra característica interesante de este análisis comparativo es que, cinco años después de la perturbación inicial, el desempleo se ubica entre 0.004 y 0.12 puntos porcentuales sobre el nivel que habría alcanzado sin dicho shock de política monetaria restrictiva. En el gráfico 3 también se observa que cuando se elimina el supuesto de que el mercado laboral de México es rígido, el efecto de la política monetaria en el desempleo es aún más pronunciado (modelo 4). La mayor persistencia estimada

GRÁFICO 3

\section{Funciones de respuesta al impulso en los cinco modelos}

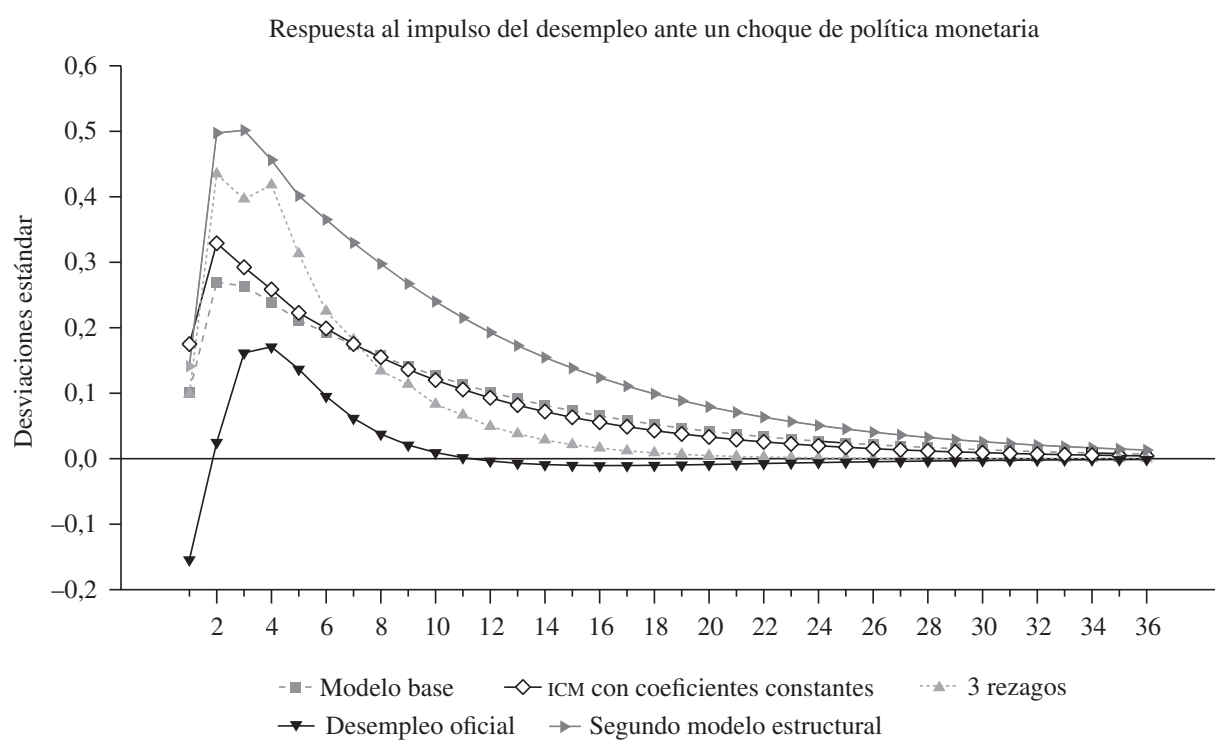

Fuente: cálculos de los autores.

Nota: modelo 1 = modelo base; modelo 2 = ICM con coeficientes constantes; modelo 3 = modelo con 3 rezagos; modelo $4=$ segundo modelo estructural; modelo 5 = desempleo oficial. 


\begin{tabular}{|c|c|c|c|c|c|c|c|c|c|c|c|c|}
\hline & \multicolumn{4}{|c|}{$\operatorname{Ln}(u)$} & \multicolumn{4}{|c|}{$\tilde{y}$} & \multicolumn{4}{|c|}{$I C M$} \\
\hline & 1 & 10 & 20 & 30 & 1 & 10 & 20 & 30 & 1 & 10 & 20 & 30 \\
\hline Modelo 1 & 93,35 & 71,71 & 69,71 & 69,50 & 5,47 & 4,28 & 4,40 & 4,42 & 1,17 & 23,99 & 25,80 & 26,07 \\
\hline Modelo 2 & 90,45 & 67,35 & 65,63 & 65,05 & 5,88 & 6,10 & 6,92 & 6,95 & 3,65 & 26,03 & 27,43 & 27,54 \\
\hline Modelo 3 & 97,87 & 74,45 & 74,55 & 74,55 & 1,21 & 3,61 & 3,99 & 3,99 & 0,95 & 21,93 & 21,45 & 21,44 \\
\hline Modelo 4 & 38,88 & 28,82 & 27,58 & 27,45 & 60,33 & 48,60 & 48,08 & 48,03 & 0,77 & 22,57 & 24,32 & 24,50 \\
\hline Modelo 5 & 88,61 & 92,14 & 92,51 & 92,51 & 9,28 & 5,54 & 5,31 & 5,30 & 2,10 & 2,29 & 2,17 & 2,17 \\
\hline
\end{tabular}

Fuente: cálculos de los autores.

Nota: las cifras de la segunda fila son horizontes (es decir, trimestres); las demás cifras son estimaciones que fueron redondeadas en dos decimales, debido a ello los errores de redondeo pueden en algunos casos impedir una descomposición porcentual perfecta.

ICM: índice de condiciones monetarias.

Ln: logaritmo natural.

$\tilde{y}$ : brecha de producto.

también se puede atribuir a la restricción impuesta de que la política monetaria no responde contemporáneamente. El modelo con la menor persistencia estimada es aquel en que se incluyen tres rezagos (modelo 3 ).

En el cuadro 3 se aprecia la descomposición de la varianza del error de predicción de los cinco modelos en horizontes de 1, 10, 20 y 30 trimestres. Como se puede constatar, la proporción de varianza del desempleo explicada por la política monetaria es bastante robusta para los modelos 1 a 4 en dichos horizontes de tiempo. Los resultados indican que en el modelo con tres rezagos, la varianza del desempleo se explica en un $21 \%$ por la política monetaria en el horizonte de 30 trimestres, comparado con el $26 \%$ que se reporta en el modelo base.

\section{VII}

\section{Conclusiones}

Se ha estimado la repercusión de las políticas macroeconómicas de estabilización en las tasas de desempleo mexicanas. El análisis se llevó a cabo respecto de dos series de tasas de desempleo (la tasa oficial y la tasa alternativa calculada según la metodología utilizada por la Encuesta Continua de Población de la Oficina de Estadísticas Laborales de los Estados Unidos de América) y bajo distintas especificaciones del modelo.

Cuando se utilizan las tasas de desempleo oficiales, la función impulso-respuesta indica que los shocks de política monetaria generan un descenso inicial del desempleo de alrededor de 0,15 puntos porcentuales. Este
El modelo 5, en que se usa la tasa oficial de desempleo, merece especial atención. Los resultados sugieren que el efecto contemporáneo de un shock de un punto porcentual de política monetaria restrictiva generaría una reducción del desempleo igual a 0.15 puntos porcentuales, y que dicho efecto alcanzaría su punto máximo luego de cuatro trimestres. La función de respuesta al impulso vuelve a cero luego de dos años y medio. El análisis de descomposición de la varianza denota que las tasas de desempleo oficiales son exógenas con respecto a los shocks de política monetaria. Por ahora, no se tiene una explicación clara para este resultado y solo se sugiere examinarlo con mayor detalle. valor aumenta rápidamente a 0,18 puntos porcentuales hacia el cuarto trimestre. Sin embargo, el desempleo vuelve luego a su nivel inicial hacia el décimo trimestre; es decir, las tasas de desempleo oficiales sugieren que el mercado laboral mexicano es muy fluido.

Cuando se utiliza la tasa de desempleo alternativa, los resultados indican que la política monetaria no es neutral en el corto y mediano plazo. Se evaluó la robustez de los hallazgos de este trabajo bajo distintos grupos de supuestos. Se llegó a la conclusión de que los resultados no dependen de ningún supuesto en particular. Por ejemplo, se observa el mismo resultado cualitativo 
cuando se trabaja con el supuesto de un mercado laboral rígido que cuando se toma como supuesto un mercado de trabajo flexible. Las funciones de impulso-respuesta sugieren que la repercusión de un shock de política monetaria en el desempleo disminuye entre 0,26 y 0,50 puntos porcentuales bajo distintos conjuntos de supuestos. Otro resultado importante es que cinco años después de la perturbación inicial, el desempleo se ubica entre 0,004 y 0,12 puntos porcentuales sobre el nivel que habría alcanzado sin el shock. En ningún caso se observa evidencia alguna de la existencia de un efecto a largo plazo de la política monetaria en el desempleo.

El análisis de descomposición de la varianza complementa los hallazgos relacionados con la función impulso-respuesta. Mientras que los modelos basados en la medida alternativa de desempleo utilizada en este trabajo sugieren que la política monetaria puede explicar hasta un $27 \%$ de la varianza total, el modelo basado en la tasa de desempleo oficial indica que la política monetaria solo puede explicar hasta un 2,2\% del total de las variaciones en el desempleo. Obviamente, estos resultados representan un enigma que requiere mayor investigación.

Los cálculos de la elasticidad del desempleo a los shocks monetarios efectuados sugieren que su efecto es bastante menor. Sin embargo, la adaptación a tales perturbaciones es más rápida, por ejemplo, que en los países europeos. La baja repercusión de la política monetaria se puede explicar por la existencia de cierta rigidez institucional, a saber, la regulación laboral intrusiva y los sindicatos de trabajadores. Estas rigideces impiden una adaptación más completa a los shocks de política. Sin embargo, existen otros elementos dentro del mercado laboral que permiten que se produzca una adaptación relativamente rápida a esas perturbaciones. Entre estos elementos, hay que considerar la existencia de un amplio sector informal y un creciente sector de servicios donde gran parte de la legislación laboral no se cumple.

APÉNDICE

CUADRO A

Estimación del modelo SVAR

(Modelo con expectativas de inflación, valores p entre paréntesis)

\begin{tabular}{|c|c|c|c|}
\hline & $\begin{array}{l}\text { Desempleo } \\
\quad \operatorname{Ln}(u)\end{array}$ & $\begin{array}{c}\text { Demanda interna } \\
\left(y-y^{*}\right)\end{array}$ & $\begin{array}{c}\text { Política monetaria } \\
\text { (ICM) }\end{array}$ \\
\hline Logaritmo natural ( $u$ ) & $\begin{array}{c}0,761 \\
(0,000)\end{array}$ & $\begin{array}{l}-0,369 \\
(0,072)\end{array}$ & $\begin{array}{c}0,837 \\
(0,133)\end{array}$ \\
\hline$(y-y *)$ & $\begin{array}{l}-0,005 \\
(0,919)\end{array}$ & $\begin{array}{c}0,562 \\
(0,000)\end{array}$ & $\begin{array}{c}0,246 \\
(0,382)\end{array}$ \\
\hline$L(I C M)$ & $\begin{array}{c}0,025 \\
(0,081)\end{array}$ & $\begin{array}{l}-0,073 \\
(0,007)\end{array}$ & $\begin{array}{c}0,689 \\
(0,000)\end{array}$ \\
\hline Producto de los Estados Unidos & $\begin{array}{l}-0,184 \\
(0,015)\end{array}$ & $\begin{array}{c}0,300 \\
(0,036)\end{array}$ & $\begin{array}{c}0,188 \\
(0,621)\end{array}$ \\
\hline$\left(q-q^{*}\right)$ & $\begin{array}{c}-0,026 \\
(0,918)\end{array}$ & $\begin{array}{c}1,894 \\
(0,000)\end{array}$ & $\begin{array}{l}3,645 \\
(0,008)\end{array}$ \\
\hline Productividad $(t)$ & $\begin{array}{c}0,105 \\
(0,869)\end{array}$ & $\begin{array}{l}-3,149 \\
(0,011)\end{array}$ & $\begin{array}{r}-15,615 \\
(0,000)\end{array}$ \\
\hline Superávit fiscal $(g)$ & $\begin{array}{c}-0,0005 \\
(0,905)\end{array}$ & $\begin{array}{c}0,004 \\
(0,563)\end{array}$ & $\begin{array}{l}-0,084 \\
(0,000)\end{array}$ \\
\hline $\operatorname{Lag} \inf \left(\pi_{1}(t-1)\right)$ & $\begin{array}{r}0,0009 \\
(0,878)\end{array}$ & $\begin{array}{l}-0,037 \\
(0,002)\end{array}$ & $\begin{array}{l}-0,021 \\
(0,498)\end{array}$ \\
\hline Tendencia & $\begin{array}{c}1,763 \\
(0,013)\end{array}$ & $\begin{array}{l}-3,107 \\
(0,020)\end{array}$ & $\begin{array}{l}34,596 \\
(0,000)\end{array}$ \\
\hline $\begin{array}{l}\text { Variable ficticia } 95 \\
R^{2} \text { ajustado }\end{array}$ & 0,831 & 0,809 & 0,869 \\
\hline
\end{tabular}

Logaritmo de la función de verosimilitud -331,18

Portmanteau (12) $107,1656(0,1411)$

Fuente: cálculos de los autores.

Nota: el cuadro contiene la suma de los coeficientes sobre los dos rezagos de cada variable; asimismo, la prueba de Wald para la significancia conjunta de ambos rezagos de cada variable. Se presenta la prueba de Portmanteau conjunta para residuos de ruido blanco.

ICM: índice de condiciones monetarias. 
CUADRO A.2

Prueba endógena de raíz unitaria para dos cambios estructurales basada en el multiplicador de Lagrange

Desempleo oficial. Modelo $\mathrm{C}: K=1, T_{\mathrm{B}_{1}}=1995: 1, T_{\mathrm{B}_{1}}=1999: 3, \mathrm{~N}=72, \lambda_{1}, \cong 0,4, \lambda_{2}, \cong 0,7$
Valores críticos $5 \%(-5,67) t_{0}=2,769$

\begin{tabular}{llllll}
\hline Parámetro & $\mu$ & $d_{1}$ & $d_{t_{1}}$ & $d_{2}$ & $\phi$ \\
\hline Estimado & $-0,294$ & 1,515 & 0,058 & 0,364 & 0,233 \\
Estadístico t & $-2,349^{*}$ & $5,030^{*}$ & 0,392 & 1,206 & $2,272^{*}$ \\
\hline
\end{tabular}

Desempleo alternativo. Modelo C: $K=1, T_{\mathrm{B}_{1}}=1995: 1, T_{\mathrm{B}_{2}}=1999: 3, \mathrm{~N}=72, \lambda_{1} \cong 0,4, t_{0},=-5,452$

Valores críticos $5 \%(-5,67) t_{0}=-5,452$

\begin{tabular}{|c|c|c|c|c|c|c|}
\hline Parámetro & $\mu$ & $d_{1}$ & $d_{t_{1}}$ & $d_{2}$ & $d_{t_{2}}$ & $\phi$ \\
\hline Estimado & $-0,574$ & 1,874 & 0,383 & 0,487 & 0,808 & $-0,6412$ \\
\hline Estadístico t & $-3,583^{*}$ & $3,062 *$ & $1,622 * *$ & 0,797 & $3,761 *$ & $-5,452$ \\
\hline
\end{tabular}

Fuente: cálculos de los autores.

Nota: *denota valores al $5 \%$ de significancia; **denota valores al $10 \%$ de significancia.

Hipótesis nula: $y_{t}=\mu_{0}+d_{1} B_{1 t}+\mathrm{d}_{t_{1}} D_{1 t}+d_{2} B_{2 t}+d_{\mathrm{t}_{2}} D_{2 t}+y_{t-1}+v_{1 t}$

Hipótesis alternativa: $y_{t}=\mu_{1}+\gamma t+d_{1} D_{1 t}+d_{t_{1}} D T_{1 t}^{2}+d_{2} D_{2 t}+d_{\mathrm{t}_{2}} D T_{2 t}+v_{2 t}$

donde $D_{j t}=1$ para $t \geq T_{B j}+1, j=1,2$ y 0 en otro caso; $D T_{j t}=t-T_{B j}$ para $t \geq T_{B j}+1, j=1,2$ y 0 en otro caso; $B_{j t}=1$ para $t \geq T_{B j}+1, j=1,2 \mathrm{y}$

0 en otro caso y $T_{B j}$ denota el tiempo en que ocurre el cambio.

Alcaraz, C. (2009), "Informal and formal labour flexibility in Mexico", Desarrollo y sociedad, № 63, Bogotá, D.C., Centro de Estudios sobre Desarrollo Económico (CEDE).

Alcaraz, C., D. Chiquiar y M. Ramos-Francia (2008), "Diferenciales salariales intersectoriales y el cambio en la composición del empleo urbano de la economía mexicana en 2001-2004", Documentos de investigación, $\mathrm{N}^{\circ}$ 2008-06, México, D.F., Banco de México.

Alexius, A. y B. Holmlund (2007), "Monetary policy and Swedish unemployment fluctuations", IZA Discussion Paper, N 2933, Bonn, Institute for the Study of Labor, julio.

Banxico (Banco de México) (2002), "Informe sobre la inflación, octubre-diciembre 2001 y Programa monetario para 2002" [en línea] http://www.banxico.org.mx/documents/\%7B15AA921B8C80-3651-02F2-B0AE5A7BB9E6\%7D.pdf

Blanchard, O. (2003), "Monetary Policy and Unemployment" [en línea] http://econ-www.mit.edu/files/731

Blanchard, O. y P. Diamond (1989), "The Beveridge curve", Brookings Papers on Economic Activity, $\mathrm{N}^{\mathrm{o}}$ 1, Washington, D.C., The Brookings Institution.

Blanchard, O. y D. Quah (1989), "The dynamic effects of aggregate demand and supply disturbances", American Economic Review, vol. 79, $\mathrm{N}^{\circ}$ 4, Nashville, Tennessee, American Economic Association.

Calderon-Madrid, A. (2000), "Job stability and labor mobility in urban Mexico: A study based on duration models and transition analysis", Research Network Working Paper, $\mathrm{N}^{\circ} \mathrm{R}-419$, Washington, D.C., Banco Interamericano de Desarrollo, noviembre.

Cantillon, R. (1755), Essay on the Nature of the Commerce in General.

Castellanos, S.G. (2000), "El efecto del 'corto' sobre la estructura de tasas de interés", Documento de investigación, $\mathrm{N}^{\circ}$ 2000-01, México, D.F., Banco de México.

Chick, V. (1983), Macroeconomics after Keynes: A Reconsideration of the General Theory, Cambridge, Massachusetts, The MIT Press.

Díaz de León, A. y L. Greenham (2000), "Política monetaria y tasas de interés: experiencia reciente para el caso de México",
Documentos de investigación, $\mathrm{N}^{\mathrm{o}}$ 2000-08, México, D.F., Banco de México.

Fisher, I. (1911), The Purchasing Power of Money, Nueva York, Macmillan.

Fleck, S. y C. Sorrentino (1994), "Employment and unemployment in Mexico's labor force", Monthly Labor Review, Washington, D.C., Oficina de Estadísticas Laborales, noviembre.

Friedman, M. (1976), Inflation and Unemployment, Nobel Memorial Lecture, Illinois, The University of Chicago, 13 de diciembre.

Galindo, L. y J. Ros (2005), "Alternatives to inflation targeting in Mexico", documento presentado en la Amherst/CEDES Conference on Inflation Targeting (Buenos Aires, 13 y 14 de mayo de 2005).

Garriga, A.C. (2010), "Objetivos, instrumentos y resultados de la política monetaria. México 1980-2010", Documentos de trabajo, No 225, México, D.F., Centro de Investigación y Docencia Económicas (CIDE).

Gaytán, A. y J. González (2006), "Structural changes in the transmission mechanism of monetary policy in Mexico: A non-linear VAR approach", Documento de investigación, № 2006-06, México, D.F., Banco de México.

Gill, I., C.E. Montenegro y D. Dömeland (eds.) (2001), Crafting Labor Policy: Techniques and Lessons Learned from Latin America, Washington, D.C., Banco Mundial.

González Anaya, J.A. (2002), "Labor market flexibility in thirteen Latin American countries and the United States: Revisiting and expanding Okun coefficients", Working Paper, $\mathrm{N}^{\circ} 136$, Stanford, Center for Research on Economic Development and Policy Reform, Universidad de Stanford, junio.

Grier, R. y K.B. Grier (2006), "On the real effects of inflation and inflation uncertainty in Mexico", Journal of Development Economics, vol. 80, No 2, Amsterdam, Elsevier.

Hayford, M.D. (2005), "Fiscal policy and national saving", Applied Economics, vol. 37, $\mathrm{N}^{\circ}$ 9, Taylor and Francis.

Heckman, J. y C. Pagés (2001), "The cost of job security regulation: evidence from Latin American labor markets", Economía, vol. 1, $\mathrm{N}^{\mathrm{o}}$ 1, Washington, D.C., Brookings Institution Press. 
Hume, D. (1985), "Of money", Essays: Moral, Political and Literary, E.F. Miller (ed.), Indianapolis, Liberty Fund. Publicado originalmente en 1752 .

Hyeon-Seung, H. (2005), "A simple test of exogeneity for recursively structural var model", Applied Economics, vol. 37, № 21, Taylor and Francis.

Keynes, J.M. (2001), Teoría general de la ocupación, el interés y el dinero, Fondo de Cultura Económica. Publicado originalmente en 1936.

Lee, J. y M. Strazicich (2003), "Minimum Lagrange multiplier unit root test with two structural breaks", Review of Economics and Statistics, vol. 85, No 4, Cambridge, Massachusetts, The MIT Press, noviembre.

Lipsey, R. (1960), "The relation between unemployment and the rate of change of money wage rates in the United Kingdom, 1862-1957: a further analysis", Economica, New Series, vol. 27, № 105.

Loboguerrero, A.M. y U. Panizza (2005), "Macroeconomic shocks, inflation and Latin America's labor market", Monetary Policy and Macroeconomic Stabilization in Latin America, R.J. Langhammer y L. Vinhas de Souza (eds.), Springer.

Maloney, W. (2009), "Mexican labor markets: protection, productivity and power", No Growth without Equity? Inequality, Interests and Competition in Mexico, Santiago Levy y Michael Walton (eds.), Washington, D.C., Banco Mundial.

Marshall, A. (2004), Labour Market Policies and Regulations in Argentina, Brazil and Mexico: Programmes and Impacts, Buenos Aires, Consejo Nacional de Investigaciones Científicas y Técnicas/Instituto de Desarrollo Económico y Social.

Martin, G. (2000), "Employment and unemployment in Mexico in the 1990s", Monthly Labor Review, Washington, D.C., Oficina de Estadísticas Laborales, noviembre.

Mendoza, A. (2003), "The inflation-output volatility tradeoff and exchange rate shocks in Mexico and Turkey", Central Bank Review, vol. 3 , $\mathrm{N}^{\circ}$ 1, Ankara, Banco Central de la República de Turquía.

Messmacher, M. y A. Werner (2002), "La política monetaria en México: 1950-2000”, Gaceta de economía, vol. 1, México, D.F., Instituto Tecnológico Autónomo de México (ITAM).
Naciones Unidas (2007), World Population Propsects: The 2006 Revision, Nueva York.

Newcomb, S. (1885), Principles of Political Economy, Nueva York, Harper.

Pastor, J. y A. Villagómez (2007), "The structural budget balance: A preliminary estimation for Mexico", Applied Economics, vol. 39, $\mathrm{N}^{\mathrm{o}}$ 12, Taylor and Francis.

Ravn, M. y S. Simonelli (2008), "Labor market dynamics and the business cycle: Structural evidence for the United States", Scandinavian Journal of Economics, vol. 109, № 4, Wiley.

Revenga, A. y M. Riboud (1993), "Unemployment in Mexico: its characteristics and determinants", Policy Research Working Paper Series, $N^{\circ}$ 1230, Washington, D.C., Banco Mundial, diciembre.

Risso, W.A. y E.J. Sánchez (2009), "Inflation and Mexican economic growth: long-run relation and threshold effects", Journal of Financial Economic Policy, vol. 1, № 3, Bingley, Emerald Group Publishing, mayo.

Sánchez, M. (2005), "The recent stabilization experience in Mexico", Cato Journal, vol. 25, № 1, Washington, D.C., Cato Institute.

Schettkat, R. y R. Sun (2008), "Monetary policy and European unemployment", Schumpeter Discussion Papers, $\mathrm{N}^{\mathrm{o}} 2008-002$, Universitätsbibliothek Wuppertal.

Shapiro, M.D. y M. Watson (1988), "Sources of business cycle fluctuations", NBER Macroeconomics Annual 1988, Stanley Fischer (ed.), Cambridge, Massachusetts, National Bureau of Economic Research.

Sims, Ch.A. (1980), "Macroeconomics and reality", Econometrica, vol. 48, No 1 , Nueva York, The Econometric Society, enero.

Sims, Ch.A., J. Stock y M. Watson (1990), "Inference in linear time series models with some unit roots", Econometrica, vol. 58, $\mathrm{N}^{\circ} 1$, Nueva York, The Econometric Society, enero.

Sims, Ch.A. y T. Zha (1999), "Error bands for impulse responses", Econometrica, vol. 67, № 5, Nueva York, The Econometric Society, septiembre.

Taylor, J.B. (1994), "The inflation/output variability trade-off revisited", Conference Series, Boston, Federal Reserve Bank of Boston. 